\title{
On Archimedean of t-norms
}

\author{
Zhang Kun-Long ${ }^{a}$ Song Li-xia ${ }^{b}$
}

Department of Basic Science , North China Institute of Science and Technology ,Beijing, 101601, China

zhk1007@126.com Songlx100@sina.com

Keywords- T-norms; Archimedean; conditional cancellation law; cancellation law; continuous.

Abstract-In this paper, we consider the Archimedean properties of t-norms. We summarize some Archimedean properties and characterize some new Archimedean properties of triangular norms on $[0,1]$.

\section{Introduction}

Triangular-norms (t-norms) and related operations (such as t-conforms, implications) play an important role e.g. in the fuzzy theory and its applications. Especially the continuous Archimedean t-norms and the continuous t-norms are popular. The latest can be built up with the help of continuous Archimedeans t-norms and the strongest t-norm, the minimum operator. Hence, examining continuous Archimedean t-norms has major importance in the field.

It is well-known from the literature that continuous Archimedean t-norms can be represented by additive generator functions [2].

Triangular norms on [0,1] were introduced in [6] and play an important role in fuzzy set theory (see e.g. $[1,7,8]$ for more details). One of the most important properties that can be satisfied by tnorms on the unit interval is the Archimedean property: continuous t-norms can be fully characterized by means of Archimedean t-norms, the Archimedean property is closely related to additive and multiplicative generators, etc. $[1,9,10]$. In this paper,we summarize some Archimedean properties and characterize some new Archimedean properties.[11].

\section{Preliminaries}

Definition 1. A triangular norm (t-norm for short)is a binary operation $\mathrm{T}$ on the unit interval [0,1],i.e.a function $T:[0,1]^{2} \rightarrow[0,1]$, such that for all $x, y, z \in[0,1]$ the following four axioms are satisfied:

(T1) $T(x, y)=T(y, x)$

(T2) $T(x, T(y, z))=T(T(x, y), z) \quad$ (associativity)

(T3) $T(x, y) \leq T(x, z)$, whenever $y \leq z \quad$ (monotonicity)

(T4) $T(x, 1)=x \quad$ (boundary condition).

Definition 2. The t-norm $\mathrm{T}$ is called Archimedean if

(AP) for each $(x, y) \in] 0, \mathbb{1}^{2}$, there is an $n \in \mathbf{N}$ with $x_{T}^{(n)}<y$.

Definition 3. The t-norm $\mathrm{T}$ satisfies the cancellation law if

(CL) $\quad T(x, y)=T(x, z)$ implies $\mathrm{x}=0$ or $\mathrm{y}=\mathrm{z}$.

Definition 4. The t-norm $\mathrm{T}$ satisfies the conditional cancellation law if

(CCL) $\quad T(x, y)=T(x, z)>0$ implies $\mathrm{y}=\mathrm{z}$.

Definition 5. The t-norm $\mathrm{T}$ has the limit property if

(lP) for all $x \in] 0,1\left[\lim _{n \rightarrow \infty} x_{T}^{(n)}=0\right.$

Definition 6. A function $\mathrm{F}:[0,1]^{2} \rightarrow[0,1]$ is continuous if for all convergent sequences $\left(x_{n}\right)_{n \in \mathbf{N}},\left(y_{n}\right)_{n \in \mathbf{N}} \in[0,1]^{\mathrm{N}}$ we have 


$$
\begin{gathered}
F\left(\lim _{n \rightarrow \infty} x_{n}, \lim _{n \rightarrow \infty} y_{n}\right)=\lim _{n \rightarrow \infty} F\left(x_{n}, y_{n}\right) \\
(x * y) *((x * y) *(y * x))=0, \forall x, y \in A .
\end{gathered}
$$

The following examples support that the four conditions in the BCK-algebra definition are independent. Therefore, in order to simplify the BCK-algebra definition we have to propose the equivalent new definition of BCK-algebra.

\section{The first characterizations to the Archimedean of t-norms}

In this paragraph,in the basis of summarizing some Archimedean properties of t-norms,we characterize some new ones and give the proofs.

Theorem 1. A continuous t-norm is Archimedean if and only if it satisfies the CCL.

Proof: A continuous Archimedean t-norm is either nilpotent or strict. Each strict and each nilpotent t-norm fulfills the CCL.Thus necessity and sufficiency are obvious.

Theorem 2. If $\lim _{x \square x_{0}} T(x, x)<x_{0}$ for each $\left.x_{0} \in\right] 0,1[$,then $T$ is Archimedean.

Proof: assume $\lim _{x \square x_{0}} T(x, x)<x_{0}$ for each $\left.x_{0} \in\right] 0,1[$.

Then because of the monotonicity (T3),

we have

$$
T\left(x_{0}, x_{0}\right) \leq \lim _{x \square x_{0}} T(x, x)<x_{0}
$$

for all $\left.x_{0} \in\right] 0,1[$, and $\mathrm{T}$ is Archimedean.

A t-norm $\mathrm{T}$ is called strict if it is continuous and strictly monotone.Thus

Theorem 3. If $\mathrm{T}$ is strict, then $\mathrm{T}$ is Archimedean.

Proof: a strict t-norm is continuous and can have only trivial idempotent elements, Theorem 3 is a special case of Theorem 2.

Therefore we have,

Theorem 4. If $\mathrm{T}$ is strictly monotone and continuous then $\mathrm{T}$ is Archimedean

Theorem 5. If each $x \in] 0,1[$ is a nilpotent element of $\mathrm{T}$,then $\mathrm{T}$ is Archimedean.

Proof: if each $x \in] 0,1[$ is a nilpotent element of $\mathrm{T}$ then $\mathrm{T}$ satisfies LP and is Archimedean.

Theorem 6. If $\mathrm{T}$ is nilpotent then $\mathrm{T}$ is Archimedean.

Proof: each nilpotent t-norm $\mathrm{T}$ is isomorphic to $T_{L}$ and $T_{L}$ is Archimedean. Obviously $\mathrm{T}$ is Archimedean.

Theorem 7. T has the limit property if and only if $\mathrm{T}$ is Archimedean.

Theorem 8. T has only trivial idempotent elements and, whenever

$$
\lim _{x \square x_{0}} T(x, x)=x_{0}
$$

for some $\left.x_{0} \in\right] 0,1\left[\right.$, there exists a $\left.y_{0} \in\right] x_{0}, 1\left[\right.$ such that $T\left(y_{0}, y_{0}\right)=x_{0}$ if and only if $\mathrm{T}$ is Archimedean.

In fact Theorem 7 is equivalent to Theorem 8. In order Wto simply the proof. we write them as follows:

For a t-non $\mathrm{T}$ the following are equivalent:

(i) $\mathrm{T}$ is Archimedean.

(ii) $\mathrm{T}$ satisfies the limit property (LP).

(iii) $\mathrm{T}$ has only trivial idempotent elements and, whenever

$\lim _{x \square x_{0}} T(x, x)=x_{0}$

for some $\left.x_{0} \in\right] 0,1\left[\right.$, there exists a $\left.y_{0} \in\right] x_{0}, 1\left[\right.$ such that $T\left(y_{0}, y_{0}\right)=x_{0}$ if and only if $\mathrm{T}$ is Archimedean. Proof: In order to show that (i) implies (iii).

Let $\mathrm{T}$ be Archimedean. The assumption that some $a \in] 0,1[$ is an idempotent element of $\mathrm{T}$ implies $a_{T}^{(n)}=a$ for all $n \in \mathbf{N}$, so T can have only trivial idempotent elements. 
If $\lim _{x \square x_{0}} T(x, x)=x_{0}$ for some $\left.x_{0} \in\right] 0,1[$ and for all $y \in] x_{0}, l\left[\right.$ we have $T(y, y)>x_{0}$ then, by induction, we also have

$y_{T}^{(n)}>x_{0}$

for all $y \in] x_{0}, l[$ and for all $n \in \mathbf{N}$, again violating (AP).

If T satisfies (iii), fix an arbitrary $x \in] 0,1\left[\right.$ and put $x_{0}=\lim _{n \rightarrow \infty} x_{T}^{(n)}$. Then, because of the monotonicity (T3), we also have

$\lim _{y \sqsubset x_{0}} T(y, y)=x_{0}$.

If $x_{0}>0$ then there is some $\left.y_{0} \in\right] x_{0}, l\left[\right.$ such that $T\left(y_{0}, y_{0}\right)=x_{0}$ and also $x_{T}^{(n)}<y_{0}$ for Some $n \in \mathbf{N}$, implying that we must have

$x_{T}^{(2 n)}=x_{0}$

for all sufficiently large $n \in \mathbf{N}$, leading to the contradiction

$T\left(x_{0}, x_{0}\right)=x_{T}^{(4 n)}=x_{0}$

Consequently, the only possibility is $x_{0}=0$. Since $\left.x \in\right] 0,1[$ was chosen arbitrarily, T satisfies (LP).

Finally, assume that $\mathrm{T}$ satisfies (ii) and choose $x, y \in] 0,1\left[\right.$. Because of $\lim _{n \rightarrow \infty} x_{T}^{(n)}=0$, there exists an $n \in \mathbf{N}$ such that $x_{T}^{(n)}<y$

$\mathrm{T}$ is Archimedean, showing that (ii) implies (i).

The logical relationship between various algebraic properties of t-norms, a double arrow indicates an implication, a dotted arrow means that the corresponding implication holds for continuous t-norms.

In addition, Archimedean t-norms also have important properties, we only give the Theorem 10, and the other proofs will be omitted:

Theorem 9. Each left-continuous cancellative Archimedean t-norm is continuous.

In fact the calculative is not necessary. Here it can be weakened as follows:

Theorem 10. Each left-continuous Archimedean t-norm is continuous.

Proof:asssume that $\mathrm{T}$ is left-continuous and Archimedean, but not right-continuous in some

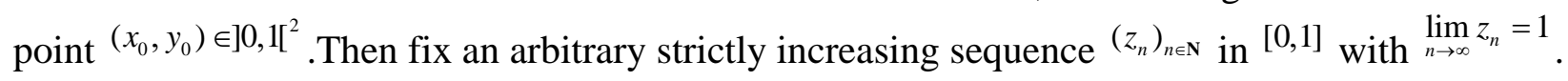

Since T is Archimedean ,for each $n \in \mathbf{N}$ there exist numbers $k_{n}, l_{n} \in \mathbf{N}$ such that

$\left(z_{n}\right)_{T}^{k_{n}} \leq x_{0}<\left(z_{n}\right)_{T}^{k_{n}-1}$,

$\left(z_{n}\right)_{T}^{l_{n}} \leq x_{0}<\left(z_{n}\right)_{T}^{l_{n}-1}$.

implying that for all $n \in \mathbf{N}$

$\left(z_{n}\right)_{T}^{k_{n}+l_{n}} \leq T\left(x_{0}, y_{0}\right)<T\left(x_{0}^{+}, y_{0}^{+}\right) \leq\left(z_{n}\right)_{T}^{k_{n}+l_{n}-2}$.

The left-continuity of T yields $\lim _{n \rightarrow \infty}\left(z_{n}\right)_{T}^{(2)}=1$ and, consequently,

$\lim _{n \rightarrow \infty} T\left(T\left(x_{0}^{+}, y_{0}^{+}\right),\left(z_{n}\right)_{T}^{(2)}\right)=T\left(x_{0}^{+}, y_{0}^{+}\right)$

But then there is some $n \in \mathbf{N}$ such that

$\left(z_{n}\right)_{T}^{k_{n}+l_{n}} \leq T\left(x_{0}, y_{0}\right)<T\left(T\left(x_{0}^{+}, y_{0}^{+}\right),\left(z_{n}\right)_{T}^{(2)}\right) \leq T\left(\left(z_{n}\right)_{T}^{k_{n}+l_{n}-2},\left(z_{n}\right)_{T}^{(2)}\right)=\left(z_{n}\right)_{T}^{k_{n}+l_{n}}$.

which is contradiction. Therefore, we have:

Theorem 11. Each cancellative Archimedean t-norm which is (left-)continuous in the point $(1,1)$ is continuous.

Theorem 12. Let $\mathrm{T}$ be a continuous Archimedean t-norm. Then the following are equivalent:

(i) $\mathrm{T}$ is nilpotent.

(ii) There exists some nilpotent element of $\mathrm{T}$.

(iii) There exists some zero divisor of $\mathrm{T}$.

(iv) $\mathrm{T}$ is not strict. 


\section{The second characterizations to the Archimedean of t-norms}

Theorem 1. $\mathrm{T}$ is right-continuous and has only trivial idempotent elements then it is Archimedean.

Proof:If a right-continuous t-norm $\mathrm{T}$ has only trivial idempotent elements then $\mathrm{T}$ satisfies (LP) by Proposition 2.6 (see [1]) and ,because of Theorem 2.12(see [1]),T is Archimedean.

It is easy to see $\mathrm{T}$ is continuous and has only trivial idempotent elements then it is Archimedean.

Theorem 2. If $\mathrm{T}$ is right-continuous and satisfies the CCL then it is Archimedean.

Proof: for an arbitrary $x \in] 0,1\left[\right.$ the sequence $\left(x_{T}^{(n)}\right)_{n \in \mathrm{N}}$ converges to some $a \in[0,1]$ which is an idempotent element of $\mathrm{T}$ because of Proposition 2.6 (see [1]). Conversely, we have $T(a, a)=T(a, 1)$ and, because of (CCL), a=0,i.e.,T has the LP and, because of Theorem 2.12 (see [1]), is Archimedean. Similarly,

Theorem 3. T is continuous and satisfies the CCL then it is Archimedean.

Theorem 4. $\mathrm{T}$ is continuous and satisfies the CL then it is Archimedean.

Proof: If t-norm T satisfies CCL,

then we can obtain $\mathrm{y}=\mathrm{z}$ by

$T(x, y)=T(x, z)>0$,

if $T(x, y)=T(x, z)=0$, then we can obtain $\mathrm{y}=\mathrm{z}$ or $\mathrm{x}=0$. Since $\mathrm{T}$ has no zero divisor , $\mathrm{x}$ can't be 0.Therefore $\mathrm{T}$ satisfies CL.

Definition 7. let $\mathrm{T}$ be a conditional calculative left-continuous t-norm which has no zero divisor, then $\mathrm{T}$ is not necessarily continuous. This example can show a conditional cancellative leftcontinuous t-norm Which is continuous in $(1,1)$ is not necessarily continuous.

Ramark 1. If a t-norm T satisfies the CL then it obviously fulfills the CCL, but not conversely. In this sense, we also obtain Theorem 4 .

Although Theorem 2 and Theorem.3 are correct for t-norms T, left-continuous conditional cancellative t-norm $\mathrm{T}$ is not necessarily Archimedean. For example, each $(x, y) \in] 0,1]^{2}$ is in a one-toone correspondence with a pair $\left(\left(x_{n}\right)_{n \in N},\left(y_{n}\right)_{n \in N}\right)$ of strictly increasing sequences of natural numbers given by the unique infinite dyadic representations.

$$
\begin{aligned}
& x=\sum_{n=1}^{\infty} \frac{1}{2^{x_{n}}} \\
& y=\sum_{n=1}^{\infty} \frac{1}{2^{y_{n}}}
\end{aligned}
$$

of the numbers $x$ and $y$, respectively .Using this notion ,then the t-norm $T:[0,1]^{2} \rightarrow[0,1]$ given by

$$
T(x, y)=\left\{\begin{array}{cc}
\sum_{n=1}^{\infty} \frac{1}{2^{x_{n}+y_{n}-n}} & (x, y) \in[0,1]^{2} \\
0 & \text { otherwise }
\end{array}\right.
$$

is strictly monotone ,therefor is calculative ,furthermore it

is conditional cancellative, left-continuous on $[0,1]^{2}$ but it is

neither Archimedean nor continuous

Theorem 5. If continuous t-norm $\mathrm{T}$ satisfies the CCL and has no zero divisor, then it is Archimedean.

All the implication between the algebraic properties of t-norms considered so far are summarized and visualized in Figure 1. Now we characterize some new properties.

Ramark 2. If $\mathrm{t}$-norm T satisfies CCL and has no zero divisor, then T necessarily satisfies CL.

Proof: If $\mathrm{t}$-norm $\mathrm{T}$ satisfies CCL, then we can obtain $\mathrm{y}=\mathrm{z}$ by $T(x, y)=T(x, z)>0$, if $T(x, y)=T(x, z)=0$, then we can obtain $\mathrm{y}=\mathrm{z}$ or $\mathrm{x}=0$.

Since $\mathrm{T}$ has no zero divisor, $\mathrm{x}$ can't be 0 .Therefore $\mathrm{T}$ satisfies CL. Thus let $\mathrm{T}$ be a conditional cancellative left-continuous t-norm which has no zero divisor, then $\mathrm{T}$ is not necessarily 
continuous.(see(*)). This example can show a conditional calculative left-continuous t-norm Which is continuous in $(1,1)$ is not necessarily continuous(This open

problem see[2]).

Theorem 6. $\mathrm{T}$ is continuous Archimedean t-norm and has no zero-divisors then $\mathrm{T}$ is strict.

Proof: Because of monotonicity, we have

$T(x, y) \leq T(x, z)$

whenever $y<z$. T has no zero divisors, so

$T(x, y) \neq 0, T(x, z) \neq 0$

we will show $T(x, y)<T(x, z)$.

if $T(x, y)=T(x, z)>0$,then $T$ satisfies the CCL. as well known ,continuous Archimedean t-norm is either nilpotent or strict. If t-norm is strict, obviously t satisfies the CL, then it satisfies the CCL.

If t-norm is nilpotent, the continuity of $\mathrm{T}$ implies that there exists a number $a \in[v, l[$ such that $y=T(z, a)$ Then

$$
\begin{aligned}
T(x, z) & =T(x, y)=T(x, T(z, a)) \\
& =T(T(x, z), a)=\cdots=T\left(T(x, z), a_{T}^{(n)}\right),
\end{aligned}
$$

for any $n \in \mathbf{N}$.because of the nilpotent of $\mathrm{T}$, we obtain $T(x, z)=0$.so $\mathrm{T}$ satisfies the CCL. so $y=z$,it violates the ${ }^{y<z}$. then $T(x, y)<T(x, z)$. e.g. $\mathrm{T}$ is strictly. Therefore $\mathrm{T}$ is strict.

\section{Conclusions}

we consider the Archimedean properties of t-norms. We summarize some Archimedean properties and characterize some new Archimedean properties of triangular norms on $([0,1], \leq)$.

\section{Acknowledgment}

This work described here is partially supported by the National Natural Science Foundation of China (19801016) (10261003) and the Fundamental Research Funds for the Central Universities (3142013021) and the North China institute of Science and Technology Key Discipline Construction Project (HKXJZD201402).

\section{References}

[1] E.P.Klement, R.Mesiar, E.Pap,Triangular Norms,Trends in Logic,Studia Logica Library, Vol.8, Kluwer, Dordrecht,2000.

[2] E.P.Klement, R.Mesiar, E. Pap,Problems on triangular norms and related operators,Fuzzy Sets and Systems 145(2004) 471-479.

[3] Funda Karacal, An answer to an open problem on triangular norms Fuzzy Sets and Systems 155(2005) 459-463.

[4] Erich Peter Klement,Radko Mesiar,Endre Pap, Triangular norms. Position paper I:Basic analytical and algebraic properties Technical Report FLLL-TR-0208

[5] P.H jek,Observations on the monodial t-norm logic,Fuzzy Sets and Systems 132(2002) 107-112.

[6] B.Schweizer, A.Sklar, Probabilistic Metric Spaces, North-Holland, NewYork, 1983.

[7] J.C.Fodor, M.Roubens, Fuzzy Preference Modelling and Multicriteria Decision Support, Kluwer Academic Publishers, Dordrecht, 1994.

[8] P.H jek, Metamathematics of Fuzzy Logic, Kluwer Academic Publishers, Dordrecht, 1998.

[9] E.P.Klement, R.Mesiar, E.Pap, Triangular norms. Position paper II: general constructions and parametrized families, Fuzzy Sets and Systems 145 (3) (2004) 411-438. 
[10] E.P.Klement, R.Mesiar, E.Pap, Triangular norms. Position paper III: continuous t-norms, Fuzzy Sets and Systems 145 (3) (2004) 439-454.

[11] Glad Deschrijver .The Archimedean property for t-norms in interval-valued fuzzy set theory ,Fuzzy Sets and Systems 157 (2006) 2311-2327. 